\title{
A multitype Hawk and Dove game
}

\author{
Aditya Aradhye ${ }^{1}$, Eitan Altman ${ }^{2}$, and Rachid El-Azouzi ${ }^{3}$ \\ ${ }^{1}$ Chennai Mathematical Institute, Chennai, India, adityaaradhye@gmail.com ${ }^{2}$ Univ \\ Côte d'Azur, INRIA, France, and LINCS, Paris, France, Eitan.Altman@inria.fr \\ http://www-sop.inria.fr/members/Eitan.Altman/ \\ ${ }^{3}$ LIA, University of Avignon, France, rachid.elazouzi@univ-avignon.fr
}

\begin{abstract}
We consider in this paper the Hawk-Dove game in which each of infinitely many individuals, involved with pairwise encounters with other individuals, can decide whether to act aggressively (Hawk) or peacefully (Dove). Each individual is characterized by its strength. The strength distribution among the population is assumed to be fixed and not to vary in time. If both individuals involved in an interaction are Hawks, there will be a fight, the result of which will be determined by the strength of each of the individuals involved. The larger the difference between the strength of the individuals is, the larger is the cost for the weaker player involved in the fight. Our goal is to study the influence of the parameters (such as the strength level distribution) on the equilibrium of the game. We show that for some parameters there exists a threshold equilibrium policy while for other parameters there is no equilibrium policy at all.
\end{abstract}

\section{Introduction}

Evolutionary games become a central tool for predicting and even design evolution in many field. The origin of evolutionary games come from biology where it was introduced by [16 to model conflicts among animals. It differs from classical game theory by (i) its focusing on the evolution dynamics of the fraction of members of the population that use a given strategy, and (2) in the notion of Evolutionary Stable Strategy (ESS, [16]) which includes robustness against a deviation of a whole (possibly small) fraction of the population who may wish to deviate (This is in contrast with the standard Nash equilibrium concept that only incorporates robustness against deviation of a single user). It became perhaps the most important mathematical tool for describing and modeling evolution since Darwin. Indeed, on the importance of the ESS for understanding the evolution of species, Dawkins writes in his book "The Selfish Gene" [19]: "we may come to look back on the invention of the ESS concept as one of the most important advances in evolutionary theory since Darwin." He further specifies: "Maynard Smith's concept of the ESS will enable us, for the first time, to see clearly how a collection of independent selfish entities can come to resemble a single organized whole." Recently, however, evolutionary game theory has become of increased interest to social scientists [8]. In computer science, evolutionary game theory 
is appearing, some examples of applications can be found in multiple access protocols [17, multihoming [14] and resources competition in the Internet [21.

In this paper we focus on the classical evolutionary Hawk-Dove game which is one of the most studied examples in evolutionary games. The Hawk-Dove game is a model for determining the degree of aggressiveness in a society in which each individual can decide on whether to be a Hawk or a Dove. There are many pairwise interactions between individuals while competing over resources such as food. While Hawkish behavior benefits an individual who meets a Dove while contending over a resource, it has a cost since it is involved in more confrontations Hawk-Hawk which are more violent and in which chances of getting wounded are high. The objective of a game analysis is then to predict what fraction of the population would be aggressive at equilibrium as a function of the system's parameters.

In this paper we assume that the choice between Dove and Hawk only determines whether or not there would be a confrontation between the individuals. But the outcome of the conflict is determined by a parameter that is proper to each of the involved individual, which we call strength. It could be related to its size, or its weight. Each individual in a large population takes a decision on whether to act aggressively (Hawk) or not (Dove) based on its own strength. It is again involved in many pairwise encounters with other individuals randomly selected from a large population. The decision to act aggressively or not is taken without knowing what will be the strength of those individuals it would meet.

In evolutionary game literature, variants of the hawk-dove game exist. For example, in [11] a dynamic version of the hawk dove game is proposed. In this version, it is assumed that each player (animal) has a state that corresponds to its level of energy reserves. A strategy of a player specifies which action to take as a function of its state. Assuming that an animal must minimize its probability of dying, the authors established a new ESS according to which an animal plays a hawk if its energy reserves are below some critical value, and plays dove otherwise. Furthermore, any single mutant that adopts other strategy than the ESS would get a strictly lower fitness. In [20, the author considered a heterogeneous population composed of two groups of hawks and doves that have different fighting abilities and which are linked via migration. Assuming that migration occurs at a much faster time scale than the game dynamics (hawk dove game), the author studied the dynamics of the full population. Other interesting extended versions of the Hawk-Dove game are proposed in [12, 3, 13, 10, 7, 5, 6, 4.

Originated in biology, the hawk-dove game lends itself well to various networking problems such as power control or medium access control as well. In 9, 2, a semi-dynamic version of the hawk-dove game applied to power control is introduced. In this game, the aggressive behavior stands for transmitting at a high power level while the peaceful behavior is associated to transmitting at a low power level. Each mobile station (player) has a state that corresponds to its energy level. The action used by a player determines its immediate fitness and its future state. Moreover, it is assumed that a player can use only the 
same strategy during its lifetime. The goal of a player is to maximize its overall amount of data sent during its lifetime. The authors identified in this context a paradox in which the fraction of a population choosing the peaceful behavior at the evolutionarily stability decreases as the initial energy state of players increases. In [1, the authors applied the hawk dove game to congestion control where the aggressive behavior corresponds to using a high-speed TCP version to be used over the Internet. Another application of the hawk-dove game in the medium access control is considered in [18.

In this paper we allow the state space to be a continuum. After presenting the model in the next section, we identify in Section 3 conditions for an equilibrium with a threshold structure to exist, in which a individual behaves aggressively if it is stronger than some threshold. We then search in Section 4 for other equilibria and show that under some conditions, any equilibrium other than threshold does not exist at all. This is due to the fact that the state space is infinite and has thus not been observed in games with finite state spaces.

\section{Model}

We consider a Hawk and Dove game, in which individuals have pairwise interactions over resources (food). Two individuals that adopt a Dove behavior split the resource peacefully; we assume that the share of each individual depends on the strength of the individual as follows. The stronger individual receives a fraction $\alpha$ of the resource and the other one receives $1-\alpha$ of it, where $\alpha$ is a constant between 0 and 1 . If it meets a individual with an aggressive behavior (Hawk) then the whole resource is taken by the aggressive individual so that the Hawk gets one unit of fitness and the Dove none.

When two Hawks meet, there is a fight in which case the true identity determines the fitness of each player. We assume that the stronger individual receives one unit of fitness whereas the weaker one's utility is monotone increasing in its strength. Let $x$ (and $y$ ) be the strength levels of the stronger (resp. weaker) individual. Then we assume that the fitness of the weaker individual is given by $-f(y, x-y)$ for some nonnegative $f$ which is assumed to be decreasing in its first argument and increasing in its second one. Each individual is encountered with another individual chosen uniformly at random. Assume that the strength level in the population is distributed according to probability density function $\theta(x)$. This means the probability that any individual encounters a individual of strength between $x$ to $x+d x$ is $\theta(x) d x$ for small $d x$. When stronger individual has strength $x$ and weaker individual has strength $y(<x)$, then payoff matrices are as follows.

The probability that a individual of strength $x$ meets another individual of strength exactly equal to $x$ is zero. Hence the payoffs in that case do not affect the utility of the individuals. 


$$
\begin{array}{|c|c|c|}
\hline & \mathrm{H} & \mathrm{D} \\
\hline \mathrm{H} & 1 & 1 \\
\hline \mathrm{D} & 0 & \alpha \\
\hline
\end{array}
$$

Table 1.*

\begin{tabular}{|c|c|c|}
\hline & $\mathrm{H}$ & $\mathrm{D}$ \\
\hline $\mathrm{H}$ & $-f(x, y-x)$ & 1 \\
\hline $\mathrm{D}$ & 0 & $1-\alpha$ \\
\hline
\end{tabular}

Table 2. *

Payoff for player with strength $x(>y)$

Payoff for player with strength $y(<x)$

We define $P$ (as a function of $x$ ) to be the strategy of the individuals. $P(x)$ is the probability of playing Hawk when at strength level $x$ (and hence probability that it plays Dove is $1-P(x))$. In general different individuals can play different strategies. But when a particular individual is encountered by a random individual, only thing that affects its utility is the probability with which it is encountered by a individual with strength $y$ and strategy Hawk and individual with strength $y$ and strategy Dove. If $h(y)$ is the probability with which the individual is encountered by a individual with strength $y$ and playing Hawk, we can equivalently assume that all individuals with strength $y$ are playing

Hawk with probability $\frac{h(y)}{\theta(y)}$. Hence forward we shall assume all the individuals in the environment play the same strategy. Define the utility of the individual as $U\left(P^{\prime}, x, P\right)$, this is the expected utility that a individual (of strength $x$ ) gets when it uses strategy $P^{\prime}$ and the rest of the population uses strategy $P$. It is given by

$$
U\left(P^{\prime}, x, P\right)=P^{\prime}(x) U(H, x, P)+\left(1-P^{\prime}(x)\right) U(D, x, P)
$$

where $H$ is pure strategy of playing Hawk and $D$ is pure strategy of playing Dove. We have,

$$
\begin{gathered}
U(H, x, P)=\int_{0}^{x} \theta(y) d y+\int_{x}^{\infty} \theta(y) P(y)(-f(x, y-x)) d y+\int_{x}^{\infty} \theta(y)(1-P(y)) d y \\
U(D, x, P)=\alpha \int_{0}^{x} \theta(y)(1-P(y)) d y+(1-\alpha) \int_{x}^{\infty} \theta(y)(1-P(y)) d y
\end{gathered}
$$

\section{Threshold Strategy}

Before studying the existence of Nash equilibrium and ESS, let us define the threshold strategy based on the strength level.

Definition 1. We define a threshold strategy $P$ by

$$
P(x)=\left\{\begin{array}{cl}
0 & \text { if } x<L \\
1 & \text { if } x>L \\
\text { any value } \in[0,1] & \text { if } x=L
\end{array}\right.
$$


We call $L$ as threshold value of this threshold strategy, and denote the threshold strategy as $P_{L}$.

Theorem 1. If $f$ is a bounded function, then there exists a threshold strategy (for $\alpha=1 / 2$ ), such that if it is used by the individual and the population, it is Nash equilibrium. If the function $f$ is strictly increasing in $x$, then this threshold strategy is also an ESS.

Proof. Part 1 - Existence of threshold strategy which is Nash equilibrium.

We find the conditions on threshold value $L$ so that its threshold strength is Nash equilibrium. Let $P_{L}$ be a Nash equilibrium. This $U\left(P_{L}, x, P_{L}\right) \geq$ $U\left(P, x, P_{L}\right)$ for every strategy $P$, and every strength level $x$. We have

$P_{L}(x) U\left(H, x, P_{L}\right)+\left(1-P_{L}(x)\right) U\left(D, x, P_{L}\right) \geq P(x) U\left(H, x, P_{L}\right)+(1-P(x)) U\left(D, x, P_{L}\right)$

Case 1: $x>L$

For $x>L$, we have $P_{L}(x)=1$, to satisfy (2), we must have

$U\left(P_{L}, x, P_{L}\right) \geq U\left(P, x, P_{L}\right)$

$U\left(H, x, P_{L}\right) \geq P(x) U\left(H, x, P_{L}\right)+(1-P(x)) U\left(D, x, P_{L}\right)$

$(1-P(x)) U\left(H, x, P_{L}\right) \geq(1-P(x)) U\left(D, x, P_{L}\right)$

Above inequality has to be satisfied by all strategies $P$, so it is necessary and sufficient that

$U\left(H, x, P_{L}\right) \geq U\left(D, x, P_{L}\right)$

$\int_{0}^{x} \theta(y) d y+\int_{x}^{\infty} \theta(y)(-f(x, y-x)) d y \geq \frac{1}{2} \int_{0}^{L} \theta(y) d y$

$\int_{0}^{x} \theta(y) d y \geq \frac{1}{2} \int_{0}^{L} \theta(y)+\int_{x}^{\infty} \theta(y) f(x, y-x) d y$

LHS of the above statement is increasing in $x$ and as $f$ is decreasing in $x$, RHS is decreasing in $x$. So, it is necessary and sufficient that inequality is satisfied for $x=L$.

$$
\begin{gathered}
\int_{0}^{L} \theta(y) d y \geq \frac{1}{2} \int_{0}^{L} \theta(y)+\int_{L}^{\infty} \theta(y) f(L, y-L) d y \\
\frac{1}{2} \int_{0}^{L} \theta(y) d y \geq \int_{L}^{\infty} \theta(y) f(L, y-L) d y
\end{gathered}
$$


Case 2: $x<L$

For $x<L$, we have $P_{L}(x)=0$, to satisfy (2), we must have

$U\left(P_{L}, x, P_{L}\right) \geq U\left(P, x, P_{L}\right)$

$U\left(D, x, P_{L}\right) \geq P(x) U\left(H, x, P_{L}\right)+(1-P(x)) U\left(D, x, P_{L}\right)$

$P(x) U\left(D, x, P_{L}\right) \geq P(x) U\left(H, x, P_{L}\right)$

It is necessary and sufficient to have $U\left(D, x, P_{L}\right) \geq U\left(H, x, P_{L}\right)$

$\int_{0}^{L} \theta(y) d y+\int_{L}^{\infty} \theta(y)(-f(x, y-x)) d y \leq \frac{1}{2} \int_{0}^{L} \theta(y) d y$

$\frac{1}{2} \int_{0}^{L} \theta(y) d y \leq \int_{L}^{\infty} \theta(y) f(x, y-x) d y$

LHS of the above statement is constant in $x$ and as $f$ is decreasing in $x$, RHS is decreasing in $x$. So, it is necessary and sufficient that inequality is satisfied for $x=L$.

$\int_{0}^{L} \theta(y) d y \leq(1 / 2) \int_{0}^{L} \theta(y)+\int_{L}^{\infty} \theta(y) f(L, y-L) d y$

$$
\frac{1}{2} \int_{0}^{L} \theta(y) d y \leq \int_{L}^{\infty} \theta(y) f(L, y-L) d y
$$

(3) and (4) imply

$$
\frac{1}{2} \int_{0}^{L} \theta(y) d y=\int_{L}^{\infty} \theta(y) f(L, y-L) d y
$$

Above equation also tells us that $U\left(H, L, P_{L}\right)=U\left(D, L, P_{L}\right)$, so a player with strength $L$ is indifferent in playing Hawk and Dove, so $P_{L}(L)$ can take any value between 0 and 1 and still $P_{L}$ will be a Nash equilibrium. So, it is sufficient for $L$ to satisfy the above equation for $P_{L}$ to be Nash equilibrium.

LHS of (5) is increasing in $L$ and RHS of (5) is decreasing in $L$. At $L=0$, LHS takes value 0 and as $L$ tends to $\infty$, LHS tends to $1 / 2$. If RHS is bounded, as $f$ is a non negative, not identically zero bounded function, RHS takes a positive value at $L=0$ and tends to 0 as $L$ tends to $\infty$. Hence, (5) has unique solution. So, we have a threshold strategy which is Nash equilibrium whenever the RHS is bounded.

Part 2 - 
For $P_{L}$ to be ESS, for all strategies $P$ other than $P_{L}$ and for all $x$ except maybe on a set of measure zero, at least one of the following must hold,

1) $U\left(P_{L}, x, P_{L}\right)>U\left(P, x, P_{L}\right)$

2) $U\left(P_{L}, x, P_{L}\right)=U\left(P, x, P_{L}\right)$ and $U\left(P_{L}, x, P\right)>U(P, x, P)$

Let $P_{L}$ be the threshold strategy we get in Part 1 which is Nash equilibrium. Then we have, $U\left(H, L, P_{L}\right)=U\left(D, L, P_{L}\right)$

Also, as for $x \geq L, U\left(D, x, P_{L}\right)=\frac{1}{2} \int_{0}^{L} \theta(y) d y$ and

$U\left(H, x, P_{L}\right)=\int_{0}^{x} \theta(y) d y+\int_{x}^{\infty} \theta(y) P(y)(-f(x, y-x)) d y$, we have

$U\left(H, x, P_{L}\right)>U\left(H, L, P_{L}\right)$ for $x>L \quad$ (Strict inequality because $f$ is strictly increasing)

So for $x>L$, we have

$U\left(H, x, P_{L}\right)>U\left(H, L, P_{L}\right)=U\left(D, L, P_{L}\right)=U\left(D, x, P_{L}\right)$, which implies

$U\left(P_{L}, x, P_{L}\right)>U\left(P, x, P_{L}\right)$

Similarly, we can also show the same for $x<L$. This proves that $P_{L}$ is also an ESS as $L$ is set of measure zero.

Threshold equilibrium for general $\alpha$ : We now try to find if there exists a threshold Nash equilibrium strategy for general $\alpha$. We assume the probability density function $\theta$ is differentiable and decreasing. We assume $f$ is bounded and decreasing in $x$. It is also reasonable to assume that $f$ is concave in the variable $x$ (meaning $\frac{\delta^{2}}{\delta^{2} x} f(x, y-x) \leq 0$ for all $x$ ).

\section{Case $1: x \geq L$}

In this case, $P_{L}(x)=1$. For $P_{L}$ to be Nash equilibrium, we must have for any other strategy $P$ and for all $x \geq L, U\left(P_{L}, x, P_{L}\right) \geq U\left(P, x, P_{L}\right)$

$$
\begin{aligned}
& \Leftrightarrow U\left(H, x, P_{L}\right) \geq P(x) U\left(H, x, P_{L}\right)+(1-P(x)) U\left(D, x, P_{L}\right) \\
& \Leftrightarrow(1-P(x)) U\left(H, x, P_{L}\right) \geq(1-P(x)) U\left(D, x, P_{L}\right) \\
& \Leftrightarrow U\left(H, x, P_{L}\right) \geq U\left(D, x, P_{L}\right) \\
& \Leftrightarrow \int_{x}^{\infty} \theta(y)(-f(x, y-x)) d y+\int_{0}^{x} \theta(y) d y \geq \alpha \int_{0}^{L} \theta(y) d y
\end{aligned}
$$


$\Leftrightarrow \int_{0}^{x} \theta(y) d y \geq \alpha \int_{0}^{L} \theta(y)+\int_{x}^{\infty} \theta(y) f(x, y-x) d y$

LHS of the above statement is increasing in $x$ and as $f$ is decreasing in $x$, RHS is decreasing in $x$. So, it is sufficient that equation is satisfied for $x=L$.

$$
\begin{gathered}
\int_{0}^{L} \theta(y) d y \geq \alpha \int_{0}^{L} \theta(y)+\int_{L}^{\infty} \theta(y) f(L, y-L) d y \\
(1-\alpha) \int_{0}^{L} \theta(y) d y \geq \int_{L}^{\infty} \theta(y) f(L, y-L) d y
\end{gathered}
$$

\section{Case 2 : $x<L$}

In this case, $P_{L}(x)=0$. For $P_{L}$ to be Nash equilibrium, we must have for any other strategy $P$ and for all $x \leq L$,

$$
\begin{aligned}
& U\left(P_{L}, x, P_{L}\right) \geq U\left(P, x, P_{L}\right) \\
& \Leftrightarrow U\left(D, x, P_{L}\right) \geq P(x) U\left(H, x, P_{L}\right)+(1-P(x)) U\left(D, x, P_{L}\right) \\
& \Leftrightarrow P(x) U\left(D, x, P_{L}\right) \geq P(x) U\left(H, x, P_{L}\right) \\
& \Leftrightarrow U\left(D, x, P_{L}\right) \geq U\left(H, x, P_{L}\right) \\
& \Leftrightarrow \alpha \int_{0}^{x} \theta(y) d y+(1-\alpha) \int_{x}^{L} \theta(y) d y \geq \int_{L}^{\infty} \theta(y)(-f(x, y-x)) d y+\int_{0}^{L} \theta(y) d y \\
& \quad \Leftrightarrow \alpha \int_{0}^{L} \theta(y) d y \leq(2 \alpha-1) \int_{0}^{x} \theta(y) d y+\int_{L}^{\infty} \theta(y) f(x, y-x) d y \quad(7)
\end{aligned}
$$

Let $F(x)$ denote the RHS of (7). LHS is independent of $x$, so (7) holds for $x<L$ if and only if $F(x)$ satisfies the inequality $L H S \leq \min _{0 \leq x \leq L}\{F(x)\}$.

$$
F(x)=(2 \alpha-1) \int_{0}^{x} \theta(y)+\int_{L}^{\infty} \theta(y) f(x, y-x) d y
$$

As $\theta$ is assumed differentiable, $F$ is twice differentiable.

$$
\begin{aligned}
& F^{\prime}(x)=(2 \alpha-1) \theta(x)+\int_{L}^{\infty} \theta(y) \frac{\delta}{\delta x} f(x, y-x) d y \\
& F^{\prime \prime}(x)=(2 \alpha-1) \theta^{\prime}(x)+\int_{L}^{\infty} \theta(y) \frac{\delta^{2}}{\delta^{2} x} f(x, y-x) d y
\end{aligned}
$$

As $f$ is concave in $x$ and $\theta$ is decreasing, $F^{\prime \prime}(x) \leq 0 \forall x \leq L$

So, $F$ takes minimum value either at 0 or $L$. 
For this case, it is necessary and sufficient that (7) is satisfied by $x=0$ and $x=L$. These with (6) are the necessary and sufficient conditions for $L$ to Nash equilibrium threshold strategy. So, $P_{L}$ is Nash equilibrium if and only if (1.) $\alpha \int_{0}^{L} \theta(y) d y=F(L)$ and (2.) $\alpha \int_{0}^{L} \theta(y) d y \leq F(0)$.

So there exists a threshold strength $L$ such that $P_{L}$ is Nash equilibrium if and only if there exists $L$ satisfying both above equations, which when rearranged can be written as follows

$$
\begin{aligned}
& (1-\alpha) \int_{0}^{L} \theta(y) d y=\int_{L}^{\infty} \theta(y) f(L, y-L) d y \\
& \alpha \leq \frac{1}{2}+\frac{\int_{L}^{\infty} \theta(y)[f(0, y)-f(L, y-L)] d y}{2 \int_{0}^{L} \theta(y) d y}
\end{aligned}
$$

As we can see, this proves that for $\alpha=1$, (8) cannot be satisfied, hence there is no solution. For $\alpha \leq \frac{1}{2}$, there always exists a Nash equilibria threshold strength. For $\frac{1}{2}<\alpha<1$, existence of a Nash equilibrium threshold strategy depends upon whether the functions $\theta$ and $f$ satisfy $(9)$.

\section{Other equilibria}

We would now try to find if there are other equlibria. For this section we shall assume the function $f$ to be only dependent on the difference between the individual's and the opponent's strength levels (earlier $f$ was dependent on the difference in strength levels and individual's strength level). We also assume $\alpha=1 / 2$. Note that if at some strength level $x$, if the individual receives more utility by playing Hawk (Dove) then it will play Hawk (Dove) with full probability in the equilibrium.

Hence, $U(H, x, P)>U(D, x, P) \Rightarrow P(x)=1$ for $P$ to be equilibrium strategy.

Similarly, $U(H, x, P)<U(D, x, P) \Rightarrow P(x)=0$

Also, $P(x)=1 \Rightarrow U(H, x, P) \geq U(D, x, P)$,

$P(x)=0 \Rightarrow U(H, x, P) \leq U(D, x, P)$,

$0<P(x)<1 \Rightarrow U(H, x, P)=U(D, x, P)$ for $P$ to be equilibrium strategy 
Lemma : Any equilibrium strategy is monotone. Formally for an equilibrium strategy $P$, for some $x_{1}$, if $U\left(H, x_{1}, P\right)>U\left(D, x_{1}, P\right)$, then $P(x)=1 \forall x \geq x_{1}$, for some $x_{2}$, if $U\left(H, x_{2}, P\right)<U\left(D, x_{2}, P\right)$, then $P(x)=0 \forall x \leq x_{2}$.

\section{Proof :}

$U(H, x, P)=\int_{0}^{x} \theta(y) d y+\int_{x}^{\infty} \theta(y) P(y)(-f(y-x)) d y+\int_{x}^{\infty} \theta(y)(1-P(y)) d y$

After simplifying, we get $U(H, x, P)=1-\int_{x}^{\infty} \theta(y) P(y)(1+f(y-x)) d y$

$f$ is increasing in $y-x$, so $f$ is decreasing in $x$. So the quantity $\int_{x}^{\infty} \theta(y) P(y)(1+$ $f(y-x)) d y$ is decreasing in $x$. So, $U(H, x, P)$ is increasing in $x$.

$U(D, x, P)=(1 / 2) \int_{0}^{\infty} \theta(y)(1-P(y)) d y$ is independent of $x$. So, the quantity $U(H, x, P)-U(D, x, P)$ is increasing in $x$.

$U\left(H, x_{1}, P\right)>U\left(D, x_{1}, P\right) \rightarrow U\left(H, x_{1}, P\right)-U\left(D, x_{1}, P\right)>0$

For $x \geq x_{1}, U(H, x, P)-U(D, x, P) \geq U\left(H, x_{1}, P\right)-U\left(D, x_{1}, P\right)>0 \rightarrow$ $P(x)=1$.

By similar reasoning, second part also holds. This completes the proof of the lemma.

Let $A_{P}=\{x \mid U(H, x, P)>U(D, x, P)\}, B_{P}=\{x \mid U(H, x, P)=U(D, x, P)\}$, $C_{P}=\{x \mid U(H, x, P)<U(D, x, P)\}$.

Because of our lemma in this section, every element in $B_{P}$ is greater than every element in $A_{P}$ and less than every element in $C_{P}$.

Let $x_{1}=\inf \left(B_{P}\right), x_{2}=\sup \left(B_{P}\right)$. Clearly, $x_{1} \leq x_{2}$

So, we have $P(x)=0 \forall x<x_{1}$ and $P(x)=1 \forall x>x_{2}$

If $x_{1}=x_{2}, P(x)$ can take non trivial value (value greater than 0 and less than 1 ) at the most one value (It may take nontrivial value at $x_{1}\left(=x_{2}\right)$ ). So the strategy is a threshold strategy.

Now assume $x_{1}<x_{2}$. For $x_{1}<x<x_{2}, 0<P(x)<1$. So, we have $U(H, x, P)=U(D, x, P)$ on the whole interval $\left(x_{1}, x_{2}\right)$. So, we can differenti- 
ate and equate the two sides.

For $x_{1}<x<x_{2}$, the utilities are

$$
\begin{aligned}
& U(H, x, P)=\int_{0}^{x} \theta(y) d y+\int_{x}^{x_{2}} \theta(y)(-f(y-x)) P(y) d y+\int_{x}^{x_{2}} \theta(y)(1-P(y)) d y+ \\
& \int_{x_{2}}^{\infty} \theta(y)(-f(y-x)) d y \\
& U(D, x, P)=(1 / 2) \int_{0}^{x_{1}} \theta(y) d y+(1 / 2) \int_{x_{1}}^{x_{2}} \theta(y)(1-P(y)) d y \\
& U(H, x, P)=U(D, x, P) \\
& \Leftrightarrow \int_{0}^{x} \theta(y) d y+\int_{x}^{x_{2}} \theta(y)(-f(y-x)) P(y) d y+\int_{x}^{x_{2}} \theta(y)(1-P(y)) d y+ \\
& \int_{x_{2}}^{\infty} \theta(y)(-f(y-x)) d y=(1 / 2) \int_{0}^{x_{1}} \theta(y) d y+(1 / 2) \int_{x_{1}}^{x_{2}} \theta(y)(1-P(y)) d y \\
& \Leftrightarrow \int_{0}^{x_{2}} \theta(y) d y-\int_{x}^{x_{2}} \theta(y)(1+f(y-x)) P(y) d y+\int_{x_{2}}^{\infty} \theta(y)(-f(y-x)) d y= \\
& (1 / 2) \int_{0}^{x_{1}} \theta(y) d y+(1 / 2) \int_{x_{1}}^{x_{2}} \theta(y)(1-P(y)) d y
\end{aligned}
$$

We have $U(H, x, P)=U(D, x, P)$ on the whole interval $\left(x_{1}, x_{2}\right)$. So, we can differentiate (with respect to $\mathrm{x}$ ) and equate the two sides. We get,

$$
\begin{aligned}
& -\left(\int_{x}^{x_{2}} \theta(y) \frac{\delta}{\delta x} f(y-x) P(y) d y\right)+\theta(x)(1+f(0)) P(x)-\left(\int_{x_{2}}^{\infty} \theta(y) \frac{\delta}{\delta x} f(y-x) d y\right)=0 \\
& \theta(x)(1+f(0)) P(x)=\int_{x}^{x_{2}} \theta(y) \frac{\delta}{\delta x} f(y-x) P(y) d y++\int_{x_{2}}^{\infty} \theta(y) \frac{\delta}{\delta x} f(y-x) d y
\end{aligned}
$$

Since $f$ is increasing in $(y-x)$, it is decreasing in $x$. So, the quantity $\frac{\delta}{\delta x} f(y-x)$ is negative. Both the terms in the RHS are nonpositive, so RHS is nonpositive. But LHS is nonnegative, so for this equation to satisfy we must have $P(x)=0 \forall x_{1}<x<x_{2}$. Contradiction, since $x$ such that $x_{1}<x<x_{2}$ belongs to $B_{P}$, and hence $P(x)>0$.

So, for $x_{1}<x_{2}$, we do not have any solution. So, there does not exist any other equilibrium strategy other than threshold strategy.

\section{Price of Stability}

In this section, we study the inefficiency caused in the objective function by imposing the condition of Nash equilibrium for certain functions $f$. Objective function here for us is the average utility of all the players. This inefficiency is quantified by price of Stability. Formally it is defined as

Price of Stability $(P o S)=\frac{\max _{P \in \mathcal{P}_{e}} A U(P)}{\max _{P \in \mathcal{P}} A U(P)}$ 
where $A U(P)$ is average utility when the whole population plays strategy $P, \mathcal{P}$ is set of all strategies for the population, $\mathcal{P}_{e}$ is set of all equilibrium strategies for the population. Thus, PoS is the ratio of best you can get using the Nash equilibrium strategies and the overall best you can get. Note, PoS is always less than or equal to 1 . We shall find $\operatorname{PoS}$ for some density functions $\theta$ and cost functions $f$. Apart from previous conditions on $\theta$ and $f$, we would generally put the restrictions on $f$ that $f(x, 0)=0$ and fix a constant, say $\frac{1}{2}$, we would generally want for fixed $x, f(x, y-x)$ tend to infinity or a constant at least $\frac{1}{2}$ as $y-x$ tend to infinity. We shall now calculate PoS for some examples with the restrictions defined.

Example 1. We consider the case where $\alpha=\frac{1}{2}$. Let $\theta(y)=\mu e^{-\mu y}$ and $f(x, y-x)=1-e^{-\mu(y-x)}$.

The value of threshold strength $L$ can be calculated by solving the equation

$\frac{1}{2} \int_{0}^{L} \theta(y) d y=\int_{L}^{\infty} \theta(y) f(L, y-L) d y$

Solving, we get $e^{-\mu L}=\frac{1}{2}$ or $L=\frac{1}{\mu} \ln (2)$.

Thus, the utility for player with strength $x<L$ is $\frac{1}{2} \int_{0}^{L} \mu e^{-\mu y} d y=\frac{1}{2}\left(1-e^{-\mu L}\right)=$ $\frac{1}{4}$

For $x>L$ it is $\int_{0}^{x} \mu e^{-\mu y} d y-\int_{x}^{\infty} \mu^{-\mu y}\left(1-e^{-\mu(y-x)}\right) d y=1-\frac{3}{2} e^{-\mu x}$

Average utility $A U=\int_{0}^{L} \mu e^{-\mu x}\left(\frac{1}{4}\right) d x+\int_{L}^{\infty} \mu e^{-\mu x}\left(1-\frac{3}{2} e^{-\mu x}\right) d x$

$$
\begin{aligned}
& =\frac{1}{4}\left(1-e^{-\mu L}\right)+e^{-\mu L}-\left(\frac{3}{2}\right)\left(\frac{1}{2}\right) e^{-2 \mu L} \\
& =\frac{1}{4}\left(1-\frac{1}{2}\right)+\frac{1}{2}-\left(\frac{3}{2}\right)\left(\frac{1}{2}\right) \frac{1}{4}=\frac{7}{16}
\end{aligned}
$$

As this is unique Nash equilibrium, it is best Nash equilibrium. It can be clearly seen that when strategies are not restricted to Nash equilibrium, the average payoff is maximized when all players play Dove, the average payoff in this case is $\frac{1}{2}$. So, PoS for this game $\frac{7}{8}$.

Example 2. Consider the same $\alpha$ and $\theta$, but $f(x, y-x)=1-e^{-c \mu(y-x)}$ for some constant $>0$. By same calculations, we can check that

$e^{-\mu L}=\frac{c+1}{3 c+1}$ and $A U=\frac{1}{2}-\frac{c(c+1)}{2(3 c+1)^{2}}$.

We cannot have $c=0$ (in that case, $\mathrm{f}(\mathrm{x}, \mathrm{y}-\mathrm{x})=0$ which is not allowed), but any $c>0$ is allowed. As $c \downarrow 0, A U \rightarrow \frac{1}{2}$ and so $P o S \rightarrow 1$. So, for any small $\varepsilon>0$, we can create a game by choosing proper value of $c$ such that PoS of this game 
is $1-\varepsilon$.

Example 3. Same $\alpha$ and $\theta, f(x, y-x)=e^{k \mu(y-x)}$ where $k \geq 0$ is a constant. We can find threshold strength only for $k<1$. So, for $k \geq 1$, there is no Nash equilibrium strategy, so by definition, PoS is zero. For $k<\overline{1}$, we get $e^{-\mu L}=\frac{1-k}{3-k}$

and $A U=\frac{1}{2}-\frac{1-k}{2(3-k)^{2}}$. As $k \uparrow 1, A U \rightarrow \frac{1}{2}$ and so $P o S \rightarrow 1$. So, again for any small $\varepsilon>0$, we can create a game by choosing proper value of $k$ such that PoS of this game is $1-\varepsilon$.

\section{References}

1. E. Altman, R. El-Azouzi, Y. Hayel, and H. Tembine. The evolution of transport protocols: An evolutionary game perspective. Computer Networks, 53(10):1751 1759, 2009.

2. E. Altman, D. Fiems, M. Haddad, and J. Gaillard. Semi-dynamic hawk and dove game, applied to power control. In Proceedings IEEE INFOCOM, pages 2771-2775, March 2012.

3. E. Altman, J. Gaillard, M. Haddad, and P. Wiecek Bio-Inspired Models of Networks, Information, and Computing Systems: 6th International ICST Conference, BIONETICS 2011, chapter Dynamic Hawk and Dove Games within Flocks of Birds, pages 115-124. Springer Berlin Heidelberg, Berlin, Heidelberg, 2012.

4. Eitan Altman and Ilaria Brunetti. Revisiting evolutionary game theory. IEEE Conference on Decision and Control (CDC) 2013: 1842-1847.

5. P. Auger and D. Pontier. Fast game dynamics coupled to slow population dynamics: A single population with hawk-dove strategies. Mathematical and Computer Modelling, 27(4):81 - 88, 1998.

6. Nesrine Ben Khalifa, Rachid ElAzouzi, and Yezekael Hayel. Evolutionary games in interacting communities. Dynamic Games and Applications Journal.

7. R. Cressman. The Stability Concept of Evolutionary Game Theory. Springer-Verlag Berlin Heidelberg, 1992.

8. E. Friedman and S. Henderson. Fairness and efficiency in processor sharing protocols to minimize sojourn times. Proceedings of ACM SIGMETRICS, pages 229-337, 2003.

9. M. Haddad, E. Altman, D. Fiems, and J. Gaillard. Paradoxes in semi-dynamic evolutionary power control game: When intuition fools you! IEEE Transactions on Wireless Communications, 12(11):5728-5739, Nov. 2013.

10. Y. Hayel, E. V. Belmega, and E. Altman. Hawks and doves in a dynamic framework. Dynamic Games and Applications, 3(1):24-37, 2012.

11. A. I. Houston and J. M. McNamara. Fighting for food: a dynamic version of the hawk-dove game. Evolutionary Ecology, 2(1):51-64, 1988.

12. J. M. McNamara and A. I. Houston. If animals know their own fighting ability, the evolutionarily stable level of fighting is reduced. Journal of Theoretical Biology, 232(1):1-6, 2005.

13. J. M. McNamara, S. Merad, and E. J. Collins. The hawk-dove game as an averagecost problem. Advances in Applied Probability, 23(4):667-682, 1991. 
14. S. Shakkottai, E. Altman, and A. Kumar. The Case for Non-Cooperative Multihoming of Users to Access Points in IEEE 802.11 WLANs. In IEEE Infocom, Barcelona, Spain, 2006.

15. J. Maynard Smith. Evolution and the theory of Games. Cambridge University Press, UK, 1982.

16. M. Smith. Game theory and the evolution of fighting. In John Maynard Smith, On Evolution (Edinburgh University Press), pp.8-28, 1972.

17. Anurag Kumar Srinivas Shakkottai, Eitan Altman. Evolutionary power control games in wireless networks". 14 Journal on Selected Areas in Communications, pages $1207-1215$.

18. H. Tembine, E. Altman, and R. El-Azouzi. Delayed evolutionary game dynamics applied to medium access control. In Proceedings of the IEEE 4th International Conference on Mobile Adhoc and Sensor Systems, pages 1-6, Pisa, Italy, oct. 2007.

19. C. C. Wright. An explanation for some aspects of the behaviour of congested road traffic in terms of a simple model. Transportation Research, 9(5):267 - 273, 1975.

20. J. Yearsley. Hawks and doves in heterogeneous environments. Mathematical and Computer Modelling, 27(4):99-108, 1998.

21. Youquan Zheng and Zhenming Feng. Evolutionary game and resources competition in the internet. In Modern Communication Technologies, 2001. SIBCOM-2001. The IEEE-Siberian Workshop of Students and Young Researchers, pages 51-54, 2001. 